\title{
Factorisation is not unique for higher dimensional knots.
}

\author{
Author(en): Bayer, Eva \\ Objekttyp: Article
}

Zeitschrift: Commentarii Mathematici Helvetici

Band(Jahr): 55(1980)

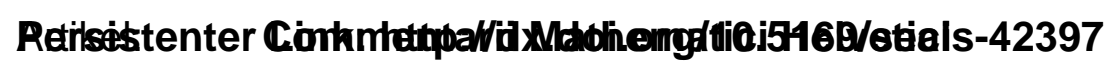

Erstellt am: May 31, 2012

\begin{abstract}
Nutzungsbedingungen
Mit dem Zugriff auf den vorliegenden Inhalt gelten die Nutzungsbedingungen als akzeptiert. Die angebotenen Dokumente stehen für nicht-kommerzielle Zwecke in Lehre, Forschung und für die private Nutzung frei zur Verfügung. Einzelne Dateien oder Ausdrucke aus diesem Angebot können zusammen mit diesen Nutzungsbedingungen und unter deren Einhaltung weitergegeben werden. Die Speicherung von Teilen des elektronischen Angebots auf anderen Servern ist nur mit vorheriger schriftlicher Genehmigung des Konsortiums der Schweizer Hochschulbibliotheken möglich. Die Rechte für diese und andere Nutzungsarten der Inhalte liegen beim Herausgeber bzw. beim Verlag.
\end{abstract}

\section{SEALS}

Ein Dienst des Konsortiums der Schweizer Hochschulbibliotheken c/o ETH-Bibliothek, Rämistrasse 101, 8092 Zürich, Schweiz

retro@seals.ch

http://retro.seals.ch 


\title{
Factorisation is not unique for higher dimensional knots
}

\author{
EVA BAYER*
}

\section{Introduction}

An $n$-knot will be a smooth oriented submanifold $K$ of the $(n+2)$-sphere $S^{n+2}$, where $K$ is homeomorphic to $S^{n}$. A knot is irreducible if it cannot be written as a connected sum of two non-trivial knots. Schubert has shown that every 1-knot can be written uniquely as a connected sum of finitely many irreducible knots (see [S] or [K1, Section 1]). For $n>2$, Sosinskii has proved that it is still possible to factorise every $n$-knot into finitely many irreducible knots (cf. [So Theorem 5. 1] or $[K 1$, Section 2]) but Kearton has shown that this factorisation is not necessarily unique for $n=3[K]$. In the present note we shall prove the non uniqueness of the factorisation for $(2 q-1)$-knots, $q \geqq 3$ and for $(2 q)$-knots, $q \geqq 4$.

I would like to thank $\mathrm{C}$. Weber for advising me to consider Levine duality in the even dimensional case. I also thank M. Kervaire for useful conversations.

\section{Factorisation is not necessarily unique for $(2 q-1)$-knots, $q \geqq 3$}

Let $q \geqq 3$ be an integer.

DEFINITION. A Seifert matrix $A$ is a square matrix of integers such that $\operatorname{det}\left(A+(-1)^{q} A^{t}\right)= \pm 1$, where $A^{t}$ is the transpose of $A$.

Let $A$ be a non-singular Seifert matrix (that is, $\operatorname{det}(A) \neq 0$ ). We shall say that $A$ is irreducible if $A$ is not $S$-equivalent to the orthogonal sum of two nonsingular Seifert matrices. (See [Le] for the definition of $S$-equivalence. In the examples that we shall construct, the Seifert matrices will be unimodular, and unimodular Seifert matrices are $S$-equivalent if and only if they are integrally congruent (see [T, Proposition 4.3])). We shall use the notation

$$
S=A+(-1)^{q} A^{t}, \quad z=S^{-1} A
$$

\footnotetext{
* Supported by a grant from the Alexander von Humboldt Foundation.
} 
LEMMA 1. Let $A_{1}$ and $A_{2}$ be Seifert matrices with $z_{1}=z_{2}$. Then

$\left(\begin{array}{cc}A_{1} & 0 \\ 0 & -A_{1}\end{array}\right)$ and $\left(\begin{array}{cc}A_{2} & 0 \\ 0 & -A_{2}\end{array}\right)$

are integrally congruent.

LEMMA 2. There exist irreducible Seifert matrices $A_{1}$ and $A_{2}$ such that

a) $z_{1}=z_{2}$

b) $A_{1}$ and $A_{2}$ are not $S$-equivalent

$A_{1}$ and $-A_{2}$ are not $S$-equivalent.

(We shall give explicit examples of such Seifert matrices after the proof of this lemma.)

The above two lemmas give the desired result. Indeed, let $A_{1}$ and $A_{2}$ be Seifert matrices as in Lemma 2.

Levine has shown that the $S$-equivalence classes of non-singular Seifert matrices correspond biunivoguely to the isotopy classes of simple $(2 q-1)$-knots [Le, Theorems 1,2,3]. Note that this implies that irreducible Seifert matrices correspond to irreducible knots. Let $K_{1}, L_{1}, K_{2}, L_{2}$ be the simple $(2 q-1)$-knots corresponding to $A_{1},-A_{1}, A_{2},-A_{2}$ respectively. These knots are irreducible, because $A_{1}$ and $A_{2}$ are irreducible. By Lemma 1 ,

$$
\left(\begin{array}{cc}
A_{1} & 0 \\
0 & -A_{1}
\end{array}\right) \text { and }\left(\begin{array}{cc}
A_{2} & 0 \\
0 & -A_{2}
\end{array}\right)
$$

are integrally congruent, as $z_{1}=z_{2}$. Therefore they are $S$-equivalent. So by [Le, Theorem 3] the connected sum of $K_{1}$ and $L_{1}$ is isotopic to the connected sum of $K_{2}$ and $L_{2}$. On the other hand, [Le, Theorem 1] shows that $K_{1}$ is not isotopic either to $K_{2}$ or to $L_{2}$, as the Seifert matrices are not $S$-equivalent.

Proof of Lemma 1. Let $A$ be a Seifert matrix, and let

$$
M_{1}=\left(\begin{array}{cc}
A & 0 \\
0 & -A
\end{array}\right), \quad M_{2}=\left(\begin{array}{cc}
0 & (-1)^{q}\left(1-z^{t}\right) \\
z & 0
\end{array}\right)
$$


Then $M_{1}$ and $M_{2}$ are integrally congruent. Indeed, let

$$
X=\left(\begin{array}{cc}
1-z & (-1)^{q} S^{-1} \\
-z & (-1)^{q} S^{-1}
\end{array}\right)=\left(\begin{array}{cc}
I & I \\
0 & I
\end{array}\right)\left(\begin{array}{cc}
I & 0 \\
0 & \left(S^{-1}\right)^{t}
\end{array}\right)\left(\begin{array}{cc}
I & 0 \\
(-1)^{q+1} A & I
\end{array}\right)
$$

where $I$ is the identity matrix.

One checks by direct computation that $M_{2}=X^{t} M_{1} X$ (it is useful to note that $1-z^{t}=A S^{-1}$, and that $\left.\left(1-z^{t}\right) A=A z\right)$. This proves Lemma 1 , as $z_{1}=z_{2}=z$,

$$
\left(\begin{array}{cc}
A_{1} & 0 \\
0 & -A_{1}
\end{array}\right) \text { and }\left(\begin{array}{cc}
A_{2} & 0 \\
0 & -A_{2}
\end{array}\right)
$$

are both congruent to

$$
\left(\begin{array}{cc}
0 & (-1)^{q}\left(1-z^{t}\right) \\
z & 0
\end{array}\right)
$$

Proof of Lemma 2. Let $\phi$ be the cyclotomic polynomial corresponding to the $15^{\text {th }}$ roots of unity. Let $t$ be the Jordan matrix associated with $\phi$, and let $z=(1-t)^{-1}$. Note that $\operatorname{det}(z)=1:$ indeed, $\operatorname{det}(1-t)=\phi(1)=1$.

Let $\zeta$ be a primitive $15^{\text {th }}$ root of unity. Sending $\zeta$ to $\zeta^{-1}$ induces a non-trivial involution on $\mathbf{Z}[\zeta]$. We shall denote this involution by an overbar.

Let $\Delta=\left\{x \in \mathbf{Q}(\zeta) \mid \operatorname{Tr}_{\mathbf{Q}(\zeta) / \mathbf{Q}}(x \mathbf{Z}[\zeta]) \subset \mathbf{Z}\right\}$ be the inverse different of the extension $\mathbf{Q}(\zeta) / \mathbf{Q}$. We have: $\bar{\Delta}=\Delta$.

DEFINITION. Let $V$ be a torsion free $\mathbf{Z}[\zeta]$-module of finite rank. We shall say that a hermitian or skewhermitian form

$$
h: V \times V \rightarrow \Delta
$$

is unimodular, if

$$
\begin{aligned}
\operatorname{ad}(h): V & \rightarrow \operatorname{Hom}_{\mathbf{z}[\zeta]}\left(V_{1} \Delta\right) \\
x & \rightarrow h(, x)
\end{aligned}
$$

is an isomorphism.

Claim 1. The integral congruence classes of Seifert matrices $A$ such that

$$
\left(A+(-1)^{q} A^{t}\right)^{-1} A=z
$$


$\left(z=(1-t)^{-1}\right.$ as above, fixed; $q$ a fixed integer $)$ are in bijection with the isometry classes of $(-1)^{q}$-hermitian unimodular forms

$$
h: \mathbf{Z}[\zeta] \times \mathbf{Z}[\zeta] \rightarrow \Delta .
$$

Proof of Claim 1. Let $A$ be a Seifert matrix with property (1), and let $S=A+(-1)^{q} A^{t}$. Rank $(A)=$ degree $(\phi)=8$. Let $V$ be a free $\mathbf{Z}$-module of rank 8 . We can consider $S$ as a $(-1)^{a}$-symmetric form $S: V \times V \rightarrow \mathbf{Z}$, and $t=$ $1-z^{-1}: V \rightarrow V$ will be an isometry for $S$.

Setting $\zeta \cdot v=t(v)$ for $v$ in $V$ makes $V$ into a $\mathbf{Z}[\zeta]$-module. As $t$ corresponds to the Jordan matrix of $\phi, V$ is isomorphic to $\mathbf{Z}[\zeta]$.

As in [B-M, §1], we associate to $S$ a $(-1)^{q}$-hermitian form

$$
h: \mathbf{Z}[\zeta] \times \mathbf{Z}[\zeta] \rightarrow \Delta
$$

such that

$$
\operatorname{Tr}_{\Phi(\zeta) / \Phi} h(\alpha x, y)=S(\alpha x, y) \forall \alpha \in \Phi(\zeta) \forall x, y \in V
$$

It is easy to check that $h$ is unimodular and that congruent Seifert matrices determine isometric $(-1)^{q}$-hermitian forms.

Conversely, given a unimodular $(-1)^{q}$-hermitian form $h: \mathbf{Z}[\zeta] \times \mathbf{Z}[\zeta] \rightarrow \Delta$, the formula (2) determines a $(-1)^{q}$-symmetric matrix $S$ such that $\operatorname{det}(S)= \pm 1$ and $t$ is an isometry for $S$. Set $A=S z$. Then $A+(-1)^{a} A^{t}=S$, therefore $A$ is a Seifert matrix" satisfying (1). Isometric $(-1)^{a}$-hermitian forms determine congruent Seifert matrices.

Claim 2. The isometry classes of unimodular $(-1)^{q}$-hermitian forms

$$
h: \mathbf{Z}[\zeta] \times \mathbf{Z}[\zeta] \rightarrow \Delta
$$

are in bijection with $U_{0} / N(U)$, where $U$ is the group of units of $\mathbf{Z}[\zeta], U_{0}$ is the group of units of $\mathbf{Z}[\zeta+\bar{\zeta}]$, and $N: U \rightarrow U_{0}, N(u)=u \bar{u}$, is the norm map.

Proof of Claim 2. Let $g$ be the minimal polynomial of $\zeta+\bar{\zeta}$, and let

$$
\alpha_{0}=\frac{1}{g^{\prime}(\zeta+\bar{\zeta})} \frac{1}{\zeta-\bar{\zeta}}
$$


Let $\Delta_{1}$ be the inverse different of the extension $\mathbf{Q}(\zeta) / \mathbf{Q}(\zeta+\bar{\zeta})$, and $\Delta_{2}$ the inverse different of $\mathbf{Q}(\zeta+\bar{\zeta}) / \mathbf{Q}$. Then $\Delta=\Delta_{1} \cdot \Delta_{2}$ [L, III. §1, Proposition 5] and

$$
\begin{aligned}
& \Delta_{1}=\frac{1}{\zeta-\bar{\zeta}} \mathbf{Z}[\zeta] \\
& \Delta_{2}=\frac{1}{g^{\prime}(\zeta+\bar{\zeta})} \mathbf{Z}[\zeta+\bar{\zeta}]
\end{aligned}
$$

[L, III. $\$ 1$ Corollary of Proposition 2]. Therefore $\Delta=a_{0} \mathbf{Z}[\zeta]$. Notice that $\bar{a}_{0}=$ $-a_{0}$.

Let $h: \mathbf{Z}[\zeta] \times \mathbf{Z}[\zeta] \rightarrow \Delta$ be a unimodular $(-1)^{q}$-hermitian form. We have: $h(x, y)=a x \bar{y}$ for some $a$ in $\Delta$ such that $\bar{a}=(-1)^{a} a$.

As we can identify $\operatorname{Hom}_{\mathbf{Z}[\zeta]}(\mathbf{Z}[\zeta], \Delta)$ with $\Delta$, the unimodularity of $h$ implies that $a \mathbf{Z}[\zeta]=\Delta$. Therefore $a \mathbf{Z}[\zeta]=a_{0} \mathbf{Z}[\zeta]$. This implies that $a a_{0}^{-1}$ is a unit. We have $\overline{a a_{0}^{-1}}=(-1)^{q+1} a a_{0}^{-1}$.

Set

$$
u= \begin{cases}a a_{0}^{-1} & \text { if } q \text { is odd } \\ a a_{0}^{-1}(\zeta-\bar{\zeta}) & \text { if } q \text { is even }\end{cases}
$$

$\zeta-\bar{\zeta}$ is a unit: $(\zeta-\bar{\zeta})^{2}(\zeta+\bar{\zeta})(-\zeta-\bar{\zeta}+1)=1$.

Therefore $u$ is in $U_{0}$ in both cases. Conversely, to $u \in U_{0}$ we associate the $(-1)^{a}$-hermitian form $h(x, y)=a x \bar{y}$ where $a$ is given by (3). One checks easily that two $(-1)^{q}$-hermitian forms are isometric if and only if the corresponding units are in the same class in $U_{0} / N(U)$.

Let us determine the cardinality of $U_{0} / N(U)$. We have

$$
\left[U_{0}: N(U)\right]=\frac{\left[U_{0}: U_{0}^{2}\right]}{\left[N(U): U_{0}^{2}\right]} .
$$

Using the theorem of Dirichlet on the rank of the group of units, we see that $\left[U_{0}: U_{0}^{2}\right]=16$.

Let $\mu$ be the group of roots of unity in $\mathbf{Q}(\zeta)$. Then

$$
\left[N(U): U_{0}^{2}\right]=\left[U: \mu U_{0}\right]=Q
$$

and $Q=2\left[\mathrm{~L} 1\right.$, Chap. 3, Theorem 4.1]. So $\left[U_{0}: N(U)\right]=8$. (We shall actually exhibit 8 distinct classes of $U_{0} / N(U)$ in the next section.) 
Applying Claim 1 and Claim 2, we see that there are 8 non-congruent Seifert matrices $A$ such that

$$
\left(A+(-1)^{q} A^{t}\right)^{-1} A=z .
$$

Therefore it is possible to choose $A_{1}$ and $A_{2}$ satisfying (1), and such that $A_{1}$ is not congruent either to $A_{2}$ or to $-A_{2}$. But congruence and $S$-equivalence are the same in this case, because the Seifert matrices are unimodular (see [T, Proposition 4.3]). $A_{1}$ and $A_{2}$ are irreducible, as their Alexander polynomial is irreducible.

\section{Explicit examples}

Let $\zeta=e^{2 i \pi / 15}$, and let $u_{1}=1, u_{2}=\zeta+\zeta^{-1}$. We have $u_{2}\left(-u_{2}^{3}+u_{2}^{2}+4 u_{2}-4\right)=1$, therefore $u_{2}$ is a unit. But $u_{2}$ is not in $N(U)$ : indeed, $u_{2}$ is conjugate to $\zeta^{7}+\zeta^{-7}$ which is negative. Clearly $-u_{2}$ is also negative, therefore not in $N(U)$. Using similar methods for the units $u_{3}=\zeta^{2}+\zeta^{-2}, u_{4}=u_{2} u_{3}=\zeta+\zeta^{-1}+\zeta^{3}+\zeta^{-3}$, we see that $u_{1},-u_{1}, u_{2},-u_{2}, u_{3},-u_{3}, u_{4},-u_{4}$ are all in different classes of $U_{0} / N(U)$. In the proof of Lemma 2 we have seen that the cardinality of $U_{0} / N(U)$ is 8 , therefore we have a complete set of representants of $U_{0} / N(U)$.

Using the method given in the proof of Lemma 2, let us associate the Seifert matrices $A_{i}$ to the units $u_{i}, i=1 \cdots 4$.

Then the $\left(\begin{array}{cc}A_{i} & 0 \\ 0 & -A_{i}\end{array}\right)$ are all different factorisations of the same Seifert matrix $B$ (see Lemma 1). Moreover, $B$ has no other factorisations than these four. Direct computation gives the following matrices for $A_{1}$ and $A_{2}$ :

q odd:

$$
A_{1}=\left(\begin{array}{rrrrrrrr}
1 & 1 & 1 & 1 & 1 & 0 & -1 & -2 \\
1 & 1 & 1 & 1 & 1 & 1 & 0 & -1 \\
1 & 1 & 1 & 1 & 1 & 1 & 1 & 0 \\
1 & 1 & 1 & 1 & 1 & 1 & 1 & 1 \\
0 & 1 & 1 & 1 & 1 & 1 & 1 & 1 \\
-1 & 0 & 1 & 1 & 1 & 1 & 1 & 1 \\
-2 & -1 & 0 & 1 & 1 & 1 & 1 & 1 \\
-2 & -2 & -1 & 0 & 1 & 1 & 1 & 1
\end{array}\right)
$$




$$
A_{2}=\left(\begin{array}{rrrrrrrr}
2 & 2 & 2 & 2 & 1 & 0 & -2 & -3 \\
2 & 2 & 2 & 2 & 2 & 1 & 0 & -2 \\
2 & 2 & 2 & 2 & 2 & 2 & 1 & 0 \\
1 & 2 & 2 & 2 & 2 & 2 & 2 & 1 \\
0 & 1 & 2 & 2 & 2 & 2 & 2 & 2 \\
-2 & 0 & 1 & 2 & 2 & 2 & 2 & 2 \\
-3 & -2 & 0 & 1 & 2 & 2 & 2 & 2 \\
-4 & -3 & -2 & 0 & 1 & 2 & 2 & 2
\end{array}\right)
$$

q even

$$
A_{1}=\left(\begin{array}{rrrrrrrr}
0 & 0 & 0 & 0 & -1 & -2 & -2 & -1 \\
0 & 0 & 0 & 0 & 0 & -1 & -2 & -2 \\
0 & 0 & 0 & 0 & 0 & 0 & -1 & -2 \\
1 & 0 & 0 & 0 & 0 & 0 & 0 & -1 \\
2 & 1 & 0 & 0 & 0 & 0 & 0 & 0 \\
2 & 2 & 1 & 0 & 0 & 0 & 0 & 0 \\
1 & 2 & 2 & 1 & 0 & 0 & 0 & 0 \\
0 & 1 & 2 & 2 & 1 & 0 & 0 & 0 \\
A_{2} & & & & & & & \\
0 & 0 & 0 & -1 & -2 & -3 & -3 & -2 \\
0 & 0 & 0 & 0 & -1 & -2 & -3 & -3 \\
1 & 0 & 0 & 0 & 0 & -1 & -2 & -3 \\
2 & 1 & 0 & 0 & 0 & 0 & -1 & -2 \\
3 & 2 & 1 & 0 & 0 & 0 & 0 & -1 \\
3 & 3 & 2 & 1 & 0 & 0 & 0 & 0 \\
2 & 3 & 3 & 2 & 1 & 0 & 0 & 0 \\
0 & 2 & 3 & 3 & 2 & 1 & 0 & 0
\end{array}\right)
$$

2. Factorisation is not necessarily unique for $(2 q)$-knots, $q \geqq 4$

Let $q \geqq 4$ be an integer. Let $\Lambda=\mathbf{Z}\left[t, t^{-1}\right]$, and let $T$ be a finitely generated $\mathbf{Z}$-torsion $\Lambda$-module such that $(1-t): T \rightarrow T$ is an isomorphism. 
DEFINITION. $L: T \times T \rightarrow \mathbf{Q} / \mathbf{Z}$ is a Levine pairing if $L$ is $\mathbf{Z}$-bilinear, nonsingular, $(-1)^{q+1}$-symmetric, such that

$$
L(t x, t y)=L(x, y) \text { for } x, y \text { in } T .
$$

In [Le 1] Levine associates to every $(2 q)$-knot $K$ a Levine pairing on the Z-torsion part $T$ of $H_{q}(\tilde{X}), \tilde{X}$ being the maximal abelian cover of $X=S^{2 q+2} \backslash K$. Isotopic knots have isometric pairings. He also shows that every Levine pairing can be realized by a simple (2q)-knot [Le 1, Theorem 13.1]. Conversely, Kojima has shown that if $H_{q}(\tilde{X})$ is finite and 2-torsion free and if $q \geqq 4$, then simple (2q)-knots having isometric Levine pairings are isotopic [Ko, Theorem 1]. Therefore, the following examples determine simple $(2 q)$-knots which factorise in more than one way:

\section{q odd}

Let $T=\mathbf{Z} / 5$, and let $t(x)=-x$ for $x$ in $T$. Then $L_{1}, L_{2}: T \times T \rightarrow \mathbf{Q} / \mathbf{Z}$ given by $L_{1}(x, y)=\frac{1}{5} x y, L_{2}(x, y)=\frac{2}{5} x y$ are Levine pairings. Clearly $L_{1}$ is not isometric either to $L_{2}$ or to $-L_{2}$. But

$$
\left(\begin{array}{ll}
3 & 2 \\
1 & 1
\end{array}\right)\left(\begin{array}{cc}
1 / 5 & 0 \\
0 & 4 / 5
\end{array}\right)\left(\begin{array}{ll}
3 & 1 \\
2 & 1
\end{array}\right)=\left(\begin{array}{cc}
0 & 1 / 5 \\
1 / 5 & 0
\end{array}\right)=\left(\begin{array}{ll}
4 & 1 \\
1 & 1
\end{array}\right)\left(\begin{array}{cc}
2 / 5 & 0 \\
0 & 3 / 5
\end{array}\right)\left(\begin{array}{ll}
4 & 1 \\
1 & 1
\end{array}\right)
$$

and the isomorphisms obviously commute with $t \oplus t$.

q even

Let $T=\mathbf{Z} / 5 \oplus \mathbf{Z} / 5, t: T \rightarrow T$ given by the matrix

$$
\left(\begin{array}{ll}
0 & -1 \\
1 & -2
\end{array}\right)
$$

and let $L_{1}, L_{2}: T \times T \rightarrow \mathbf{Q} / \mathbf{Z}$ be the Levine pairings given by the matrices

$$
\left(\begin{array}{cc}
0 & 1 / 5 \\
4 / 5 & 0
\end{array}\right) \text { and }\left(\begin{array}{cc}
0 & 2 / 5 \\
3 / 5 & 0
\end{array}\right)
$$

respectively.

$L_{1}$ is not $\Lambda$-isometric either to $L_{2}$ or to $-L_{2}$. Indeed, suppose that $L_{1}$ is $\Lambda$-isometric to $\varepsilon \cdot L_{2}$, for $\varepsilon=+1$ or -1 , and let $X$ be the matrix corresponding to this isometry. Then $\operatorname{det}(X)=2 \varepsilon$. 
Let

$$
X=\left(\begin{array}{ll}
a & b \\
c & d
\end{array}\right)
$$

The relation $t X=X t$ implies $\operatorname{det}(X)=(a+b)^{2}$. But $(a+b)^{2}=2 \varepsilon$ is impossible. $L_{1} \oplus-L_{1}$ and $L_{2} \oplus-L_{2}$ are both $\Lambda$-isometric to

$$
\left(\begin{array}{cccc}
0 & 0 & 0 & 2 / 5 \\
0 & 0 & 3 / 5 & 0 \\
0 & 2 / 5 & 0 & 0 \\
3 / 5 & 0 & 0 & 0
\end{array}\right)
$$

the isometries are given by

$$
\left(\begin{array}{rr}
I & I \\
-I & I
\end{array}\right)=\left(\begin{array}{cc}
I & I \\
0 & I
\end{array}\right)\left(\begin{array}{cc}
I & 0 \\
2 I & I
\end{array}\right)\left(\begin{array}{cc}
2 I & 0 \\
0 & I
\end{array}\right)
$$

and

$$
\left(\begin{array}{ll}
3 I & I \\
2 I & I
\end{array}\right)=\left(\begin{array}{ll}
I & I \\
0 & I
\end{array}\right)\left(\begin{array}{cc}
I & 0 \\
2 I & I
\end{array}\right)
$$

respectively, with $I=\left(\begin{array}{ll}1 & 0 \\ 0 & 1\end{array}\right)$

\section{REFERENCES}

B-M E. BAYER and F. MiChEL, Finitude du nombre des classes d'isomorphisme des structures isométriques entières, Comment. Math. Helv. 54 (1979), 378-396.

K C. Kearton, Factorisation is not unique for 3-knots, Indiana Univ. Math. Jour. 28 (1979), 451-452.

K1 The factorisation of knots (preprint).

Ko S. Kojima, Classification of simple knots by Levine pairings, Comment. Math. Helv. 54 (1979) 356-367.

L S. LANG, Algebraic number theory, Addison-Wesley Publishing Company

L1 - Cyclotomic fields, Springer-Verlag (1978).

Le J. LeVINE, An algebraic classification of some knots in codimension two, Comment. Math. Helv. 45 (1970), 185-198.

Le1 Knot modules, Trans. of the A.M.S. 229 (1977), 1-50.

S H. SCHuBERT, Die eindeutige Zerlegbarkeit eines Knotens in Primknoten, S.-B. Heidelberger Akad. Wiss. Math.-Natur. Kl. 1, 3 (1949), 57-104. 
So A. B. SosinskiI, Decomposition of knots, Math. USSR Sbornik 10 (1970), 139-150.

T H. F. Trotter, Homology of group systems with applications to knot theory, Ann. of Math. 76 (1962), 464-498.

Mathematisches Institut der Universität Münster

Federal Republic of Germany

Current address:

Université de Genève, Section de Mathématiques

2-4 rue du Lièvre

1211 Genève 24

Switzerland

Received May 15, 1980. 